\title{
3. Desafíos que enfrentan la planeación y el diseño de programas de estudios a nivel superior bajo la modalidad virtual ${ }^{8}$
}

\author{
Víctor Daniel Escalante Huitrón \\ Maricela Cuéllar Orozco \\ Valentín Buján Tinoco
}

\section{Introducción}

E n la actualidad, lo que rige al mundo es la sociedad del conocimiento, lo que impacta en las estructuras y en los procesos de la reproducción material y simbólica de la sociedad está tan impregnado de operaciones de conocimiento cruzados por tecnologías que la educación obviamente no escapa, ya sea en los modos tradicionales, ya sea en los modos virtuales. La sociedad del conocimiento implica que las tecnologías de la información y de la comunicación (TIC) abren un universo de conocimientos que se ponen a disposición de la población; a las instituciones educativas les corresponde hacer un uso experto, cuya validez depende de la verificación de la producción, distribución y reproducción del conocimiento.

Dentro de este contexto, a la educación virtual se la puede entender como un modo de democratizar las oportunidades de acceso a este derecho, en un mundo que exige sistemas educativos que respondan a necesidades de educación a través de toda la vida. En este sentido, la educación virtual, de acuerdo con Quesada (2002), también considera el uso de la tecnología respecto de cómo puede facilitar y satisfacer oportunidades educativas, dondequiera que estén ocurriendo, haciéndolas más accesibles con mayor calidad y reforzando la

8 Los autores agradecen al Instituto Politécnico Nacional de México, por el apoyo otorgado para la realización de este trabajo, ya que las experiencias adquiridas en la modalidad virtual se derivan de la práctica docente (SGE, SEPI ESIA UZ, CIECAS y CECyT 2), de los trabajos de investigación (SIP: 20170793) y de la consulta a expertos que laboran en este Instituto.

Víctor Daniel Escalante Huitrón. Doctor en Ciencias Sociales en el área de Sociedad y Educación. Profesor investigador del Instituto Politécnico Nacional en la SEPI ESIA Zacatenco y en el CIECAS. e-mail: vehuitron@hotmail.com

Maricela Cuéllar Orozco. Doctora en Ciencias en Administración en el área de Instituciones de Educación Superior. Profesora investigadora del Instituto Politécnico Nacional en la en la SEPI ESIA Zacatenco. e-mail: mcuellaro@ipn.mx

Valentín Buján Tinoco. Doctor en Ciencias en Administración en el área de Instituciones de Educación Superior. Profesor investigador del Instituto Politécnico Nacional en el CECyT n. ${ }^{\circ}$ 2. e-mail: vbujan@ipn.mx 
eficacia y eficiencia en procedimientos de entrega y acceso a la información para el proceso de enseñanza-aprendizaje.

\section{Planteamiento del problema}

La evolución de la informática, las telecomunicaciones, las redes electrónicas y la tecnología han propiciado el auge de la presencia de las tecnologías de la información y la comunicación en los procesos de enseñanza-aprendizaje dentro de un contexto de globalización, donde destacan el modo de educación virtual o a distancia en los programas de educación superior.

No obstante este auge, existen instituciones educativas prestigiadas en América Latina que no han incursionado en estos sistemas. En la segunda mitad de la década de 1990, incursionan Brasil, Colombia, Costa Rica, Venezuela, Puerto Rico, Chile y Perú, con desarrollos donde se ve la oferta de cursos en línea, programas académicos formales, portales y plataformas virtuales, además de utilizar las TIC digitales como transporte de contenidos de docencia, comienzan a emplearlas en servicios administrativos de registro, administración académica, información y bienestar comunitario y, en algunos casos, se desarrolla la utilización de bibliotecas digitales, así como elementos de realidad virtual (IESALC, 2006). Actualmente, estos desarrollos se ven altamente diferenciados en todos los países de América Latina, sin embargo, se muestra ya la consolidación de algunas instituciones a distancia tanto públicas como privadas, en cuanto a tamaño, niveles de concentración, marcos normativos y lógicas de los sistemas locales (Rama, 2012).

En la actualidad, en el Instituto Politécnico Nacional de México (IPN), existen experiencias aisladas y mínimas en esta materia; por ejemplo, de acuerdo con la Dirección de Educación Superior del IPN, en el nivel superior solo existen seis programas virtuales o semipresenciales (IPN, 2014).

Por tanto, el desafío para las instituciones de educación superior es analizar la viabilidad para que incorporen sus planes de estudio a los sistemas de enseñanza-aprendizaje a distancia, acorde con las necesidades particulares de sus carreras. Una vez dictaminada su viabilidad, es necesario planear y diseñar los programas de estudios correspondientes. El propósito de este trabajo es abrir alternativas de consulta y reflexión para las instituciones que se han quedado rezagadas de este tipo de enseñanza.

La tarea no es simple, no se trata de llevar la escuela tradicional en su modalidad presencial al modo virtual, cambiando simplemente las actividades del docente y del educando 
en el aula a una actividad similar, pero remota. Tampoco se trata de arrastrar los vicios o rezagos que puedan estar presentes en la instrucción presencial.

La educación virtual implica una ardua tarea de planeación, donde se pueda prever el diseño o rediseño de los programas de estudios centrados en el trabajo autónomo del educando bajo las premisas de aprender a aprender, a ser, a hacer, a interactuar y a emprender. Con una concepción integral que incluya:

- Programas de estudio flexibles, innovadores y actualizados basados en el aprendizaje, que permitan una sólida formación académica y valores éticos del estudiante, forjen seres humanos capaces, conscientes, responsables, abiertos al cambio y que respondan a las necesidades de la sociedad.

- Un adecuado uso de las TIC, con posibilidades de formación en el nivel superior.

- Docentes experimentados y capacitados en materia didáctica y en el uso de tecnologías.

- Docentes que impulsen la educación como facilitadores.

- Un campus virtual consolidado, basado en el uso intensivo de las TIC, sustentado en los paradigmas de su modelo educativo y en la generación de nuevos ambientes de aprendizaje.

- Nuevos mecanismos de evaluación que aseguren el reconocimiento social amplio de la calidad de los servicios ofrecidos por la institución.

- Servicios educativos flexibles, multi-, inter- y transdisciplinarios, así como polivalentes y modernos procesos administrativos y académicos regulados para una actuación eficiente y eficaz.

- Procesos de gestión y gobierno que apoyen adecuadamente el cumplimiento de las funciones sustantivas de la institución.

Para que todo esto sea posible, el reto por enfrentar es disponer de los recursos financieros y de los cuadros mutidisciplinarios especializados. Una barrera es la falta de crecimiento en el ingreso de los países en desarrollo, la cual se encuentra asociada a una brecha en la productividad y a la incapacidad de América Latina y del Caribe de seguir el paso en la adopción de nuevas tecnologías en sus procesos productivos y a la lenta actualización de las destrezas. Para cerrar esta brecha, no basta con la simple importación de la última tecnología, sino que hay que conseguir que el nivel educativo y de destrezas de la población sea adecuado para explotar todo su potencial productivo (Ferrati et al., 2002).
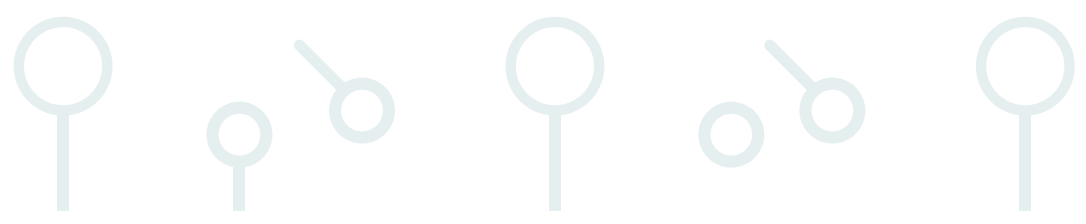


\section{Pregunta de investigación}

Lo antes expuesto permite al presente estudio ser guiado por la siguiente pregunta: ¿Qué desafíos enfrentan la planeación y el diseño de programas de estudios a nivel superior para un adecuado aprendizaje en la modalidad virtual?

\section{Método}

El método de trabajo de este estudio es de tipo documental, es decir, basado en fuentes secundarias, apoyado en la técnica de elaboración de fichas. Los pasos para realizar el estudio fueron planeación del estudio, recopilación de la información en fichas, organización y análisis de la información, redacción de borrador y documento final.

Con ello, se construyó el marco referencial teórico, contextual y organizacional de este estudio. La fuente contextual y organizacional se apoya en los documentos de trabajo del cuerpo académico que implementó la Maestría en Docencia Científica y Tecnológica en la modalidad no escolarizada y en los lineamientos de trabajo de la Unidad de Tecnología Educativa y de la Celda de Producción del Campo Virtual, áreas del Centro de Investigaciones Económicas Administrativas y Sociales, del Instituto Politécnico Nacional.

El carácter de este estudio es descriptivo, ya que destaca las particularidades de los desafíos que enfrenta la planeación y el diseño de programas de estudios a nivel superior para un adecuado aprendizaje en la modalidad virtual en tres vertientes:

\section{Desafíos de la planeación del programa de estudios}

- Fundamentación teórica

- Objetivo del programa

- Plan de estudios

- Definición de recursos tecnológicos para la operación

- Roles y requisitos del docente

Desafíos de la planeación didáctica de las unidades de aprendizaje

- Encuadre

- Planeación de la unidad

Desafíos del diseño de las unidades de aprendizaje

- Diseño de la unidad de aprendizaje

- Incorporación de la unidad a la plataforma 
En el análisis de información, se puso la atención en el examen del contenido de los documentos, en su significado, asi como en las fuentes y en su autoridad. El objetivo fue el reconocimiento de los desafíos, la planeación y el diseño de unidades bajo la modalidad virtual.

\section{Resultados}

\section{Desafíos de la planeación del programa de estudios}

\section{a. Fundamentación teórica}

Para abordar los desafíos que enfrentan la planeación y el diseño de programas de estudios, se parte de dos ámbitos: uno corresponde a los fundamentos de la planeación estratégica y táctica y otro al paradigma en el que sustenta el modelo educativo del programa de estudios.

\section{b. Planeación estratégica y táctica}

El reto en la planeación estratégica es que se conduzca como instrumento para prever y proponer caminos de actuación (modelos), a la vez que permite seleccionar y ordenar recursos, para facilitar el logro de las metas propuestas (Bris, 2002).

Que contenga un enfoque prescriptivo y normativo, de acuerdo con Mintzberg, "la planeación estratégica se refiere a la forma por la cual una organización pretende aplicar una determinada estrategia para alcanzar los objetivos propuestos" (citado en Chiavenato, 2006, p. 203).

La planeación debe contener dos premisas: el plan para proyectar y la información para desarrollar la implantación de planes. En el plan, el desafío se presenta en la formulación de la misión y visión del programa de estudios, en congruencia con los correspondientes de la institución de educación superior a la que pertenece. El reto corresponde a la identificación y el análisis de fortalezas, oportunidades, debilidades y amenazas (FODA) que enfrentará el plan (Steiner, 2007),

Una vez establecidas las premisas, existen dos momentos de la planeación estratégica: uno corresponde a la formulación y el otro a la implementación. En la formulación, se identifican las estrategias maestras (misión, visión, objetivos y políticas) y las estrategias programadas (táctica y operacional). "En esta parte de la planeación nos dedicamos a los fines más importantes y fundamentales buscados" (Steiner, 2007, p. 26) por la institución y las estrategias esenciales para lograrlos. 
En la programación táctica (de mediano plazo), se formulan planes específicos para mostrar cómo llevar a cabo las estrategias para el logro o el avance en la misión, visión y objetivos de la institución (a largo plazo). En este mismo orden de ideas, a la programación operacional (de corto plazo) le corresponde la planeación de las unidades de aprendizaje del programa de estudios.

\section{c. Programa de estudios}

Se conforma de acuerdo con el paradigma pedagógico que lo sustenta. Antes de reconocer estos paradigmas, hay que esclarecer el concepto; para ello, un referente obligado es Thomas Samuel Kuhn, quien señala que la ciencia es elaborada en el seno de una comunidad científica y no individualmente; la comunidad sirve de base a los desarrollos científicos mediante la elaboración o asunción de un paradigma del cual se derivan reglas que fijan las regularidades. El paradigma es un contexto de validez, en este caso, para respaldar el modelo educativo en el que se sustenta el programa de estudios en su modalidad virtual (Kuhn, 2004).

De acuerdo con lo anterior, y siguiendo a Terigi y Diker (1997), se tiene que buscar respuesta a algunas de las siguientes preguntas: ¿Cómo transformar la escuela moderna concebida hace trescientos años en una institución que responda a las necesidades de un mundo globalizado dentro de un mercado de trabajo flexibilizado cuyas demandas formativas se modifican constantemente? ¿Cómo respetar las diferencias culturales a través de una institución cuya estructura es profundamente homogeneizante? Así, se podría continuar con una gran cantidad de interrogantes. A cada una de ellas, se le tendría que sugerir un sustento paradigmático; por ejemplo, paradigmas acordes con el uso de las tecnologías en la escuela moderna, con los requerimientos tecnológicos y la modernidad del mercado de trabajo y con la pluralidad cultural dentro de un contexto homogeneizante. Las concepciones educativas de cada paradigma sustentan la relación pedagógica.

Para abordar el tema paradigmas dentro del contexto pedagógico, es necesario precisar cuál fundamenta el modelo educativo que soporta el plan de estudios, pudiendo ser, entre otros, conductista, humanista, cognitivo, sociocultural, constructivista o bien sustentado en competencias. Es aquí donde el desafío se centra en el estudiante como el destinatario y principal usuario, por ello, es necesario un análisis de los modelos educativos más representativos con el objetivo de que den la pauta para el diseño del plan de estudios a distancia con el carácter didáctico y autodidáctico requerido. 


\section{d. Objetivo del programa}

Los desafíos que enfrenta la formulación de objetivos del programa radican en la presencia de la base tecnológica que lo sustenta, en la desterritorialización del acto educativo que enfrenta, la congruencia de las interfaces del modelo educativo-pedagogía-didáctica-conocimientos expertos-conocimientos informáticos-recursos tecnológicos, la promoción de la expansión de la oferta y la cobertura de los servicios de educación virtual y en propiciar el interés de los estudiante en una educación superior apoyada en el uso de las TIC, la modelación y la simulación.

\section{e. Plan de estudios}

Las características de un plan de estudios bajo la modalidad virtual enfrentan el desafío de mantener las siguientes características, las cuales se apoyan en los citados por la Secretaría de Educación Superior, Ciencia y Tecnología de Santo Domingo, República Dominicana (2006):

- Pertinencia social: capacidad de respuestas a necesidades de formación de calidad que demanda la sociedad, favoreciendo la equidad en el acceso y la igualdad de oportunidades.

- Relevancia: abierta a las necesidades del entorno, tiene como opción preferente a los grupos que, cumpliendo con los requerimientos académicos correspondientes, confrontan dificultades de acceso a la educación superior y a la formación continua.

- Flexibilidad: organizar los servicios y los programas curriculares atendiendo a necesidades específicas para facilitar los aprendizajes y responder a demandas particulares de la sociedad, superando las limitaciones de espacio y tiempo. Además, facilita la incorporación de avances científicos y tecnológicos que actualizan el currículo.

- Innovación y creatividad: incorpora diversidad de prácticas educativas centrando el proceso formativo en el estudiante, su aprendizaje y su autodesarrollo.

- Autoaprendizaje: formación de competencias intelectuales para el autoestudio, el aprendizaje autónomo, el trabajo independiente y autorresponsable favorecido por la posibilidad de personalizar el proceso enseñanza-aprendizaje y adecuarlo al ritmo del estudiante,

- Comunicación: comunicación bidireccional y mediada, ya que en el proceso educativo el estudiante está fuera de la presencia del profesor. En ese sentido, se apoya en múltiples mecanismos y medios de comunicación que facilitan, estimulan y enriquecen el proceso de aprendizaje.

- Tecnologías: amplía el acceso a los diferentes sectores interesados, favorece el do- 
minio de las TIC que se requieren en la vida cotidiana y en el trabajo y reduce los costos de los programas educativos.

- Ampliación de la cobertura: ofrece mayores posibilidades de acceso a conocimientos y fuentes de información.

\section{f. Definición de recursos tecnológicos para la operación}

Incluyen, en general, plantillas para elaboración de contenido, foros, chats, cuestionarios y ejercicios tipo múltiple-opción, verdadero/falso y respuestas de una palabra. También contienen blogs y RSS. Los servicios proporcionados en general contienen control de acceso, elaboración de contenido educativo, herramientas de comunicación y la administración de grupos de estudiantes.

Mediante plataformas virtuales, tales como e-ducativa, Moodle, Agora Virtual, WebCT, Blackboard, permitir el acceso a:

- Programa de estudios y a las unidades de aprendizaje.

- La información administrativa, horarios de las sesiones, los detalles de prerrequisitos y correquisitos, información de créditos por conseguir y cómo procurar ayudas en línea.

- Publicar información actualizada de la unidad de aprendizaje.

- Registro del estudiante, seguimiento y control de actividades.

- Materiales didácticos básicos.

- Recursos adicionales, que incluyen materiales de lectura y enlaces a recursos externos, como bibliotecas digitales e internet.

- Autoevaluaciones y evaluaciones que pueden ser guardadas de forma automática.

- Procedimientos formales de evaluación.

- Ayuda de la comunicación electrónica como correo electrónico, foros y chats con moderador o sin él.

- Acceso diferenciado tanto para los instructores como para los estudiantes.

- Elaboración de documentación y estadísticas sobre el desarrollo de la unidad de aprendizaje en el formato requerido por la administración y el control de calidad institucional.

- Herramientas de ayuda que se caracterizan por ser intuitivas para crear los documentos que incluyen la inserción de imágenes e hipervínculos.

- Uso de procesadores de textos estándares u otro software para elaborar documentos.

- Uso de software especializado por área del conocimiento de la educación superior.

- Acceso a repositorios nacionales e internacionales. 
Comunicación, redes, aprendizaje y desarrollo institucional y social

\section{g. Roles y requisitos del docente}

El desafío del docente es cumplir con el papel de facilitador, orientador o guía, es fundamental para el desarrollo de un curso en la modalidad virtual. Por la naturaleza de la comunicación que se establece en los cursos virtuales, el rol de facilitador es evidente, los estudiantes no están ante un profesor que les provee toda la información, sino que ejercen un papel activo en el proceso.

El reto que distingue a la educación virtual, y que determina el aprendizaje, es la retroalimentación. En las teorías del aprendizaje conductuales y cognoscitivas, la retroalimentación desempeña un papel crucial. Toda conducta del ser humano tiene un efecto sobre el contexto, tanto físico como social. El aprendizaje tiene lugar cuando nuestras conductas y procesos mentales cambian en función de la retroalimentación que reciben del medio (Salgado, 2005).

El docente necesita plantear preguntas generadoras sobre creencias, juicios y situaciones alternativas en los contenidos de las unidad de aprendizaje y proponer actividades donde haya retroalimentación, aprovechando la plataforma virtual con dinámicas grupales con réplicas y con el fomento de la interacción y el trabajo grupal entre los compañeros y generando discusión crítica (Salgado, 2005). Asimismo, requiere orientar y redirigir las preguntas y discusiones, dar el ejemplo y aclarar de manera fundamentada las dudas que surjan y establecer normas claras de evaluación, por ejemplo, la aplicación de rúbricas.

\section{Desafíos de la planeación didáctica de las unidades de aprendizaje}

\section{a. Encuadre}

Los retos que enfrenta el encuadre se dan en la comunicación que se tiene que establecer con el educando para dar respuesta clara y concisa a los siguientes puntos de la unidad de aprendizaje: bienvenida, programa sintético, agenda de actividades, introducción, metodología, competencias, tabla de evaluación, guía del estudiante y tipos de evaluación.

En este encuadre, el desafío es dar respuestas a las siguientes preguntas: ¿Qué se va aprender? ¿Cómo se va a aprender? ¿Para qué se va a aprender? ¿Cómo se va a abordar la temática de los contenidos? ¿Cómo se va a evaluar? ¿En qué plataforma se va a trabajar? ¿Cuáles son los calendarios para el inicio y la entrega de actividades? ¿Cuáles son los tiempos para la retroalimentación y las correcciones a que se tenga lugar? ¿A quién recurrir cuando se necesita ayuda? 


\section{b. Planeación de la unidad}

La planeación parte de los datos particulares de identificación de la unidad de aprendizaje, donde se precisa su ubicación, número de horas, número de créditos (Tepic y Satca [Sistema de Asignación y Transferencia de Créditos Académicos]), profesor responsable de la planeación, entre otros datos.

El desafío se presenta en:

- Darle congruencia a la intención educativa del programa de estudios con el propósito establecido para la unidad de aprendizaje.

- Identificar en cada unidad los contenidos, los conocimientos, las habilidades y las actitudes que se proponen alcanzar, así como el porcentaje que le corresponde al estudiante dedicar al desarrollo de actividades de la unidad de aprendizaje con respecto al total del resto de unidades que estudia en paralelo, con el propósito de identificar el tiempo que se le tiene que dedicar y el peso de la calificación que le corresponde.

- Establecer los criterios de evaluación y acreditación, tales como la diagnóstica, la formativa, la sumativa y los normativos.

- Identificar y obtener las referencias que se deben poner a disposición del educando y en proponer las de apoyo complementario.

- Elaborar el plan de trabajo de acuerdo con las unidades temáticas.

- Definir la estrategia didáctica.

- Establecer las técnicas y actividades de aprendizaje y los materiales de apoyo al aprendizaje.

- Mostrar las evidencias o producto por entregar.

- Las acciones requeridas en caso de necesitar un periodo de recuperación para el educando que no logre acreditar la unidad de aprendizaje.

Actividades de control de la planeación que implican la revisión y validación de la planeación de la unidad de aprendizaje por parte de las áreas competentes. 
Comunicación, redes, aprendizaje y desarrollo institucional y social

\section{Desafíos del diseño de las unidades de aprendizaje}

\section{a. Diseño de la unidad de aprendizaje}

El reto es desarrollar, de acuerdo con la naturaleza de la unidad de aprendizaje y de manera congruente y acorde con su planeación didáctica específica, los siguientes aspectos:

- Introducir y comunicar al educando en cada apartado de la unidad de aprendizaje conceptos, teorías, métodos y prácticas para que realice las lecturas y consultas necesarias a fin de desarrollar las actividades correspondientes.

- Fomentar el pensamiento crítico relacionado con la capacidad para pensar de forma sistemática, científica, cuestionando el sustento empírico y lógico de lo que vemos, leemos o escuchamos (Facione, 1998); dichas actividades deberán estar encaminadas al fomento del pensamiento crítico de tal manera que se conciba la formación de manera racional y reflexiva, interesada en decidir qué hacer o creer (Ennis, 1985).

- Usar herramientas para la búsqueda de información apoyada en navegadores, bases de datos, metabases, software especializado, bibliotecas electrónicas, sitios web, entre otros.

- Identificar las estrategias de aprendizaje pertinentes con la unidad temática, por ejemplo, lluvia de ideas, organizadores previos, lista de cotejo, lectura de comprensión, cuadro sinóptico, diaporamas, mapa conceptual, mapa mental, organizadores gráficos, cuestionarios, diagramas, discusiones en foros, trabajos en wiki, esquemas, analogías, fichas de trabajo, ensayo, informe escrito, informe de investigación, reporte de lectura, reseña de libro, sintesis, análisis, resumen, crucigramas temáticos, así como cronometrar los tiempos que consumirá el educando, de tal forma que le resulte viable cumplir con la entrega de evidencias o productos de la unidad temática.

- Dimensionar los tiempos para la entrega de evidencias o productos diferenciando los preliminares de los definitivos.

- Identificar herramientas de comunicación y de trabajo, tales como correo electrónico, bitácoras digitales o weblogs, páginas web gratuitas, foros, áreas de anuncio, servicios de mensajería instantánea, café internet, grupos, chat, buzón digital.

\section{b. Incorporación de la unidad a la plataforma}

El reto se da en la comunicación que se establece entre los requerimientos planeados y diseñados de cada unidad de aprendizaje del programa de estudios por parte del docente con la revisión que realizan los expertos pedagogos de este trabajo para ver que se cumpla con estrategias didácticas, formas de comunicación, instrucciones, desarrollo de competencias y mecanismos de evaluación de manera pertinente. 
Una vez concluida esta actividad, nuevamente se presenta el desafío entre la comunicación que establece el docente, pero ahora con los expertos informáticos para subir a la plataforma: el encuadre, las agendas de actividades, la guía del estudiante, las referencias y los contenidos de la unidad de aprendizaje, con el propósito de que tenga la estructura lógica solicitada y que los enlaces abran los documentos o espacios de trabajo correspondientes, también que las actividades cuenten con los vínculos solicitados para foros, wikis, cuestionarios, y los que considere el docente.

\section{Discusión}

Es innegable que en el contexto educativo se encuentra la incorporación acelerada de las TIC en las aulas bajo sistemas presenciales, pero en la modalidad virtual el proceso de enseñanza-aprendizaje se basa en el desarrollo de las TIC, pero con un alto grado de complejidad, ya que no se puede avanzar si no se logra la integración requerida por las interfaces presentes en la planeación y el diseño de los programas de estudios en el nivel superior, lo cual implica la convergencia del modelo educativo-pedagogía-didáctica-conocimientos expertos-conocimientos informáticos-recursos tecnológicos. Por ello, transitar hacia la clase virtual conlleva modificar la visión del trabajo educativo, sin perder la función del aprendizaje bajo los fundamentos didácticos.

El aprendizaje del educando no se logrará si este no lo visualiza como un proyecto personal de trabajo, el cual debe identificar los conocimientos requeridos por el mercado laboral y la sociedad y que al mismo tiempo responda a los interrogantes para construir estructuras cognitivas. La dinámica de la clase cambia, ya que el docente tiene que dar atención remota y personalizada al estudiante, lo cual implica que no puede atender a la misma cantidad de estudiantes como lo hacía en la modalidad presencial. Cada paso que da el docente debe estar perfectamente planeado tanto en la propuesta estratégica como en la táctica, el avance debe estar calendarizado y el cumplimiento debe darse de manera responsable y sin excusa ni pretexto. De ahí la importancia de la comunicación y el diálogo entre los propios estudiantes y con el docente, del intercambio de ideas y de las exposiciones de razones y argumentos.

Lo anterior implica un reto para el docente, quien debe estar formado y actualizado, aparte de su especialidad, en pedagogía, en educación a distancia, en el manejo de las TIC y en el dominio del modelo educativo de la institución a la que pertenece. Se ve obligado a responder a las condiciones y necesidades que experimentan los estudiantes. Le corresponde diseñar contenidos, descripción y abordaje de estos, andamiajes, trabajos colaborativos, discusiones críticas, entre otros muchos, que están obligados a tener la virtud de la claridad 
y la factibilidad para su realización en tiempo y forma. No se le puede exigir al estudiante el cumplimiento de actividades que no cuentan con el tiempo suficiente para su realización, ni se le puede exigir al docente la revisión, retroalimentación y evaluación de actividades de un número de estudiantes que sobrepasan las posibilidades de su cumplimento para el logro de la función educativa.

El entorno del ambiente virtual debe estar apoyado por un equipo de asesores informáticos en temas de TIC, software, hardware, comunicación, pedagogía para facilitar los trabajos de planeación, diseño y operación de la modalidad virtual.

El desafío institucional radica en la gestión de las autoridades para disponer de los recursos presupuestales suficientes para llevar a cabo la implantación y operación de esta modalidad; en los aspectos normativos, deberán contar con una regulación ágil y expedita para la operación y administración escolar; sobre las TIC, disponer de los recursos tecnológicos que permitan y faciliten la operación y administración de la modalidad.

\section{Referencias}

Bris, M. N (2002). Planificación de centros educativos. Organización y calidad. Bilbao, España. Praxis.

Chiavenato, A. (2006). Introducción a la teoría general de la administración (7. a ed.). México: McGraw-Hill.

Ennis, R. H. (1985). A logical basis for measuring critical thinking skills. Educational Leadership, 43(2), 44-48.

Facione, P. A. (1998). Critical thinking: What it is and why it counts. San Francisco: Academic Press.

Ferranti, D. de, Perry, G. E., Gill, I., Guasch, J. L., Maloney, W. F., Sánchez-Páramo, C. y Schady, N. (2002). Cerrar la brecha en educación y tecnología. Washington: Banco Mundial. Recuperado de http://www-wds.worldbank.org/external/default/WDSContentServer/ WDSP/IB/2004/03/23/000160016_20040323100910/Rendered/PDF/25834010spanish010paper.pdf

IESALC (Instituto Internacional de la UNESCO para la Educación Superior en América Latina y el Caribe) (2006). Informe sobre la educación superior en América Latina y el Caribe. 2000-2005. La metamorfosis de la educación superior. Caracas: ISALC.

IPN (Instituto Politécnico Nacional) (2014). Informe de autoevaluación 2014. México: IPN.

Kuhn, T. S. (2004). La estructura de las revoluciones científicas. México: Fondo de Cultura Económica. 
Quesada, J. F. (2002). Enseñanza a distancia a través de Internet: el proyecto THALESCICA y la herramienta DELTA para el diseño electrónico de libros. http://tecnologiaedu.us.es/ edutec/paginas/151.htm

Rama, C. (2012). La reforma de la virtualización de la universidad: el nacimiento de la educación digital. México: Universidad de Guadalajara. Recuperado de http://virtualeduca. org/documentos/observatorio/libro_la-reforma-de-la-virtualizacion-de-la-universidad-claudio-rama-udg-2012.pdf

Salgado García, E. (2005). Estrategias de enseñanza virtual universitaria. Recuperado de http://www.academia.edu/2216159/Estrategias_de_ense\%C3\%B1anza_virtual_ universitaria

SEESCYT (Secretaría de Educación Superior, Ciencia y Tecnología) (2006). Reglamento de instituciones y programas de educación superior a distancia. Recuperado de https:// goo.gl/xgxfyh

Steiner, G. A. (2007). Planeación estratégica. Lo que todo director debe saber. México: Grupo Editorial Patria.

Terigi, F. y Diker, G. (1997). La formación de maestros y profesores: hoja de ruta. Buenos Aires: Paidós. 